\title{
On the trade-off between energy saving and number of switchings in green cellular networks
}

\author{
Marco Dolfi ${ }^{1}$ | Cicek Cavdar ${ }^{2}$ | Simone Morosi $^{1}$ | Pierpaolo Piunti ${ }^{1}$ | Jens Zander ${ }^{2}$ | \\ Enrico Del $\mathrm{Re}^{1}$
}

${ }^{1}$ Department of Information Engineering, University of Florence, Florence, Italy

${ }^{2}$ Communication Systems Department, KTH

Royal University of Technology, Kista,

Sweden

Correspondence

Simone Morosi, Department of Information

Engineering, University of Florence, Via di

Santa Marta, 3, 50139 Florence, Italy.

Email: simone.morosi@unifi.it

\begin{abstract}
Cellular networks are optimized by targeting multiple objectives. Usually, the different objectives are not coherent: minimizing the transmit power; the number of base station (BS) sleep-mode switchings, ie, ACTIVE/SLEEP state transitions; and the activity of the BSs and guaranteeing the quality-of-service (QoS) of users. Hence, suitable trade-offs have to be managed by network planners to provide an efficient solution to the challenge of booming mobile data. In this paper, we propose a multiobjective optimization framework aimed at minimizing the power consumption and the number of BS sleep-mode switchings in cellular networks, by jointly considering QoS requirements. These requirements are expressed in terms of a required bit rate for each mobile terminal. The framework deals with network management, such as the number of BSs that should be switched on, considering common diurnal patterns of the traffic demand. The optimization technique proposed in this paper is mixed-integer quadratic programming, which solves the joint power allocation and user association problem while also considering optimized bandwidth allocation schemes. The trade-off between the conflicting objectives, as well as the performance analysis in terms of the throughput and energy consumption of the network, is shown for different traffic load cases. The proposed optimization can obtain up to $60 \%$ energy savings during off-peak hours, guaranteeing QoS target requirements. By optimizing the network configuration, a 70\% reduction in BS switch on/off operations can be reached in a day with $3 \%$ more energy expense.
\end{abstract}

\section{1 | INTRODUCTION AND STATE OF THE ART}

The electricity consumption of telecommunication networks is forecasted to increase exorbitantly by $150 \%$ from 20 TWh in 2011 to $50 \mathrm{TWh}$ in 2020. The biggest growth is expected for mobile networks due to the immense growth of mobile data traffic by a factor of 30 caused by the more intensive use of mobile internet services. ${ }^{1}$ These are enabled by the more capable mobile networks (Long-term Evolution [LTE] and LTE Advanced technologies) and an increasing number of mobile devices with significant computing power (smartphones and tablets).

In order to gain extra commercial benefits and reduce operating expense, mobile operators are more and more motivated to seek "green" approaches that reduce the energy consumption of mobile cellular networks. Therefore, it is essential to consider how to decrease the energy consumption, especially the energy consumption of base stations (BSs), to fulfil the technical goals of future networks, such as higher user data rates, improved coverage with uniform user experience, and reduced end-to-end latency. 
The goal of improving energy efficiency (EE) and reducing the operational costs of a cellular network puts forward a large variety of critical objectives, often coupled in a conflicting manner. Since the proper design of future networks is characterized by the increasing diversity in requirements and use cases, we need to focus on the communication infrastructure and improve the versatility, scalability, and adaptability of current networks. These challenges can be addressed by developing a precise and specific problem formulation to design new optimization tools that can flexibly handle multiple objectives, trade-off analysis, and adaptive designs.

Recent studies have explored adaptive radio resource management solutions to save energy and improve network utilization efficiency. When the spatial traffic distribution is nonuniform and time varying, dynamic coverage management may be introduced to exploit traffic variations. The dynamic switch on/off of coverage overlaid cells in low traffic is an example. By this solution, the BS activity could be adapted to the traffic demand, avoiding waste of energy due to peak dimensioning. ${ }^{2-5}$ In particular, ultradense BS deployment makes sleep-mode operations more desirable, by exploiting small coverage areas and more random traffic patterns. For traffic fluctuations between day and night, macro sleep or deeper sleep can be enabled. However, for medium-to-high traffic hours, deep sleep is not possible. Therefore, in the time domain, a finer granularity of sleep mode has been introduced, identified as cell discontinuous transmission (DTX). ${ }^{6}$ This solution is enabled by the particular structure of the LTE frame and allows transceiver deactivation (sleep mode) during the idle time slots. Thus, cell coverage is not affected by the sleep mode, since the signaling symbols are always transmitted at the same power level. The cell DTX feature does not require specific decisions to be taken regarding when to sleep and traffic offloading. ${ }^{6-8}$ However, the savings that can be achieved are limited with respect to deep long-term sleep. For the long-term sleep, to maximize the energy savings, the association between users and BSs can be considered as an optimization variable together with the bandwidth allocated to each user. ${ }^{9}$ In fact, energy savings can be introduced by increasing the bandwidth per user and, consequently, reducing the BS transmission power if the target data rate per user is fixed. ${ }^{10}$ Such a solution is known as bandwidth expansion mode (BEM) and can be applied when resource usage is light, ie, in low-load conditions.

In the works of Marsan et al, ${ }^{11,12}$ analytical models are developed to identify optimal fixed BS switch-off times as a function of the daily traffic pattern. According to the authors, the extra energy savings gained by multiple switch-offs over a single switch-off is only marginal. Dynamic sleep-mode schemes generally require more switching operations as compared to fixed schemes, especially with highly variable traffic patterns. Therefore, a fundamental trade-off to be considered is that between more energy savings in sleep mode and the cost of switching operations, which includes extra power for monitoring and switching, overhead, delay constraints, and the impact on the operational lifetime of BSs. One common problem with current research in this area is that most studies implicitly assume that BSs are able to alternate between sleeping and active modes as frequently as possible. Although the most recent BSs have already been designed for frequently entering sleep modes, still, most of the current BSs in use today were designed foreseeing only occasional change of state; otherwise, the negative impact in terms of the failure rate of BS components would be dramatic.

It is worth mentioning that BS sleeping might negatively impact the quality-of-service (QoS) requirements of the system because of decreasing capacity, unless specific remedial actions are adopted concurrently. ${ }^{13}$ Nonetheless, because sleep-mode techniques are based on current architecture, they have the advantage of being easier to test and implement as no replacement of hardware is required and the performance can be evaluated by computer simulation. A potential deficiency of existing studies using this approach is oversimplified models and assumptions. ${ }^{14}$ Power control is a radio resource management solution for energy saving, which aims at minimizing the BS transmission power with a given QoS target for the served users. The benefit of power control is not only in energy reduction but also in neighbor cell interference management. ${ }^{15,16}$ Venturino et al ${ }^{15}$ proposed an optimization framework to maximize the quantity of transmitted bits per energy unit. Such a solution looks at EE maximization but does not consider a QoS target for each user. Moreover, in the work of Wong et al, ${ }^{16}$ the transmission power for an orthogonal frequency-division multiplexing communication is minimized without considering any constraint on the power model.

By combining some of these approaches, different optimization strategies have been proposed. A pricing algorithm is evaluated in the work of Yildiz et al ${ }^{17}$ to solve the user association problem and minimize area power consumption, also considering interference; therein, mobile terminal rates are fixed, and bandwidth is equally allocated to users regardless of their received signal strength. User association is a fundamental aspect of sleep-mode strategies: it implies associating mobile end users with BSs in an energy-efficient way. Users originally connected to BSs that went asleep need to be associated with new active BSs, ensuring the QoS requirements during BS sleeping operations. It is worth noticing that simply associating a user to the closest BS may be suboptimal with random traffic distribution. ${ }^{18,19}$ In the work of Holtkamp et al, ${ }^{20}$ the power supply per LTE frame is minimized by assigning a suitable transmission power and rate for each link, without considering the mapping problem and a minimum rate value to ensure QoS. Quality-of-service constraints are considered in the works of Huang et al, ${ }^{21}$ Xiao et al, ${ }^{22}$ and Ren et al. ${ }^{23}$ In the work of Huang et al, ${ }^{21}$ the power allocation per user is optimized, aiming at energy-per-bit minimization with a bit error rate constraint to guarantee QoS. A lower bound on the user data rate is set in the work of Xiao et al, ${ }^{22}$ where BS 
transmission power and user rate are optimized to maximize the EE in terms of "bits per joule". The system model considers a single-cell scenario where the effects of neighboring interference and user mapping are not considered. In the work of Ren et $\mathrm{al}^{23}$ a 2-step algorithm aiming at minimizing energy consumption by properly assigning subcarriers and power to users is proposed. Such a solution considers a very simple bandwidth division among served users and does not take advantage of BEM to reduce the unfairness in the perceived data rate.

In general, there is no available closed-form expression to show the direct relation between transmit power and QoS and user experience measures, such as service latency or user-perceived throughput. Therefore, the investigation of simplified but approximate models is accomplished to provide insights for practical system design. On the other hand, user scheduling and resource allocation solutions are needed to control the operation point that maximizes network power efficiency while balancing the heterogeneous QoS requirements. The different requirements or objectives cannot be treated separately, since they are coupled, often in conflicting ways, so that improvements in one objective end up impairing the others. These aspects call for design and optimization frameworks that handle multiple objectives and support the selection of the best attainable operating point. ${ }^{24-29}$

In this study, an energy-efficient and adaptive cellular network configuration strategy with QoS requirements is investigated: a given service rate is guaranteed to mobile terminals; if sufficient bandwidth resources are available, mobile users can obtain higher rates than the target value since their received power must be greater than the terminal sensitivity threshold. The cost of rearranging the network when traffic demand changes is taken into account by considering the number of BS switchings, defined as the number of active/inactive state transitions in a 24-hour period. In order to design an effective BS switching mechanism, 2 issues must be addressed, namely, the user association problem and BS operation. The BS on/off switching is coupled with the user association problem. In developing the energy-efficient user association mechanism, the adopted approach aims to balance the trade-off between network energy consumption and the number of switching operations, while accounting for the user target QoS constraints. This case assumes a multiobjective optimization problem with a weighting factor. When the weighting factor is equal to 0 , the user is associated to the BS that maximizes the network EE performance. As the weighting factor increases, the user association decision pays more attention to the network operation and maintenance costs, in terms of switching operations. In the simulation scenario, the traffic demand varies during the hours of a day: the problem of network dimensioning with multihour traffic is addressed by determining how much capacity is needed to handle demands at all times. Furthermore, the bandwidth blocks are not uniformly assigned but according to the spectral efficiency of the overall user associations, saving more resources for the users experiencing lower signal quality and including the benefits provided by BEM. The derived optimization framework is based on mixed-integer quadratic programming (MIQP): within this framework, the user association problem is iteratively solved, together with the decision variables for the BS activity. Moreover, the user bandwidth, the rate assignments, and the transmit power of each active BS are determined. Adapting the network configuration during the day with a minimum number of switching operations allows reducing signaling traffic and handover operations. Since the implementation of a power saving strategy should consider multiple conflicting objectives, this work extends the results presented in the work of Piunti et al ${ }^{19}$ by introducing a multiobjective optimization framework designed to manage the network configuration over a daily pattern of traffic demands. In order to present the trade-off and performance study, a per-"traffic load" class analysis is introduced, considering 3 traffic conditions: low, medium, and high.

This paper is organized as follows. Section 2 describes the system model and the considered scenario. In Section 3, the optimization problem is formulated. Section 4 describes the adopted power saving strategies, and Section 5 discusses the simulation results. Finally, Section 6 concludes the work.

\section{2 | THE SYSTEM MODEL}

A typical LTE system deployment is considered with a given bandwidth and a set of resource blocks as represented in Figure 1. A set of omnidirectional BSs provides radio access to a certain number of user equipments (UEs), as determined by a daily profile of traffic demands. ${ }^{30}$ Each BS must allocate the available bandwidth resources among the associated transmitting users by assigning the proper amount of physical resource blocks (PRBs) and guaranteeing their QoS in terms of bit rate on an hourly basis. The UEs request a constant bit rate and are served at the same time. Let $\mathcal{B}=\left\{B S_{1}, \ldots, B S_{N}\right\}$ and $\mathcal{U}=\left\{U E_{1}, \ldots, U E_{M}\right\}$ be, respectively, the set of $N$ deployed BSs and the set of $M$ users that have to be served. The binary variable $x$ models the association between BSs and UEs, as in the following:

$$
x_{i j}=\left\{\begin{array}{ll}
1, & \text { if UE } j \text { is served by BS } i \\
0, & \text { otherwise }
\end{array} i \in \mathcal{B}, j \in \mathcal{U} .\right.
$$




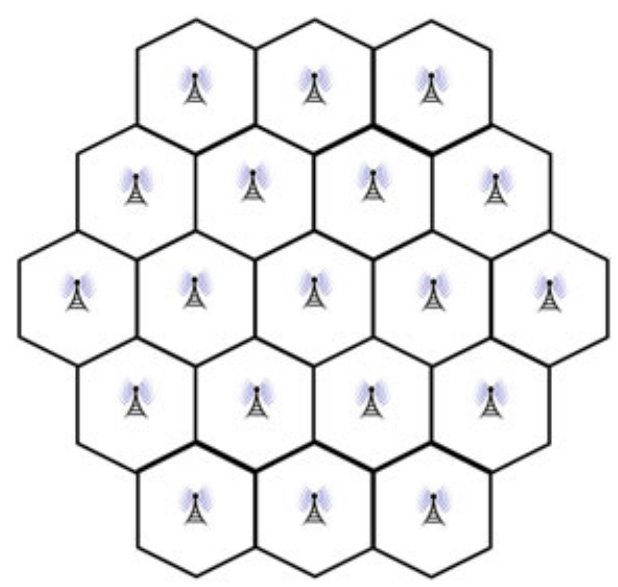

FIGURE 1 The considered BS deployment

Assuming $\pi_{i j}$ is the power assigned for transmission between BS $i$ and UE $j$ and $w_{i j}$ is the bandwidth assigned by BS $i$ to UE $j$, the data rate achieved by $\mathrm{UE} j$ is

$$
\rho_{j}=\sum_{i \in \mathcal{B}} x_{i j} w_{i j} \log _{2}\left(1+\gamma_{i j}\right)
$$

where $\gamma_{i j}$ is the signal-to-interference-plus-noise ratio (SINR) experienced by UE $j$ served by BS $i$. The transmission power of each BS $i$ is calculated as $P_{i}=\sum_{j \in \mathcal{U}} \pi_{i j} x_{i j}$. Therefore, the SINR $\gamma_{i j}$ is

$$
\gamma_{i j}=\frac{\pi_{i j} \sigma_{i j} x_{i j}}{\frac{w_{i j}}{W}\left(\sum_{k=1}^{N} P_{k} \sigma_{k j} \zeta_{k}\left(1-x_{k j}\right)+W N_{0}\right)},
$$

where $\sigma_{i j}$ is the channel loss between BS $i$ and UE $j, W$ is the total available bandwidth at the BS, and $N_{0}$ is the additive white Gaussian noise spectral density. The activity status of each BS is modeled by the binary variable $\zeta$, such that

$$
\zeta_{i}=\left\{\begin{array}{ll}
1, & \text { if BS } i \text { is active } \\
0, & \text { if BS } i \text { is in SLEEP mode }
\end{array} \quad i \in \mathcal{B} .\right.
$$

Note that in Equation 3, the received interference is weighted by the effective fraction of bandwidth assigned to the UE. Such a choice is justified by the need to consider the effect of allocating different portions of bandwidth to each UE.

\section{3 | PROBLEM FORMULATION}

Given the system defined in Section 2 and the data reported in Table 1, the goal of the problem is to minimize the global power consumption $P_{c}$ by limiting, at the same time, the number of BS switchings $S$ during daily traffic variations, reflected in the change of state of the deployed BSs and monitored by $\zeta$.

Let $\mathcal{T}=\left\{t_{1}, \ldots, t_{L}\right\}$ be the set of $L$ traffic demands during the day in terms of UEs to be served. At every time $t \in \mathcal{T}$, the 2 objective functions are then calculated as

$$
\begin{aligned}
P_{c}^{(t)} & =\sum_{i=1}^{N}\left[\left(a \sum_{j=1}^{M} \pi_{i j} x_{i j}+P_{0}\right) \zeta_{i}^{(t)}+\left(1-\zeta_{i}^{(t)}\right) P_{\text {sleep }}\right], \\
S^{(t)} & =\sum_{i=1}^{N}\left[\zeta_{i}^{(t)}\left(1-\zeta_{i}^{(t-1)}\right)+\zeta_{i}^{(t-1)}\left(1-\zeta_{i}^{(t)}\right)\right],
\end{aligned}
$$

where parameters $a, P_{0}$, and $P_{\text {sleep }}$ are the slope of the dynamic consumption, the fixed consumption, and the sleep-mode consumption, respectively. ${ }^{31}$ Considering the activity status transitions of each BS in response to the changing traffic demands, Equation $5 \mathrm{~b}$ keeps track of the number of sleep-mode switchings triggered by EE policies. 
TABLE 1 Given data for the considered problem

\begin{tabular}{ll} 
Parameter & Value \\
\hline$N$ & Number of deployed BSs \\
$M$ & Number of users \\
$\mathcal{B}=\left\{B S_{1}, \ldots, B S_{N}\right\}$ & Set of $N$ deployed BSs \\
$\mathcal{U}=\left\{U E_{1}, \ldots, U E_{M}\right\}$ & Set of $M$ users that have to be served \\
$R_{t}$ & Data rate target for each UE \\
$P_{\mathrm{MIN} j}$ & Sensitivity of UE $j$ \\
$P_{\mathrm{MAX}}$ & Maximum allowed BS transmission power \\
$N_{p}$ & Number of available PRBs at the BS \\
$W_{p}$ & Bandwidth of a single PRB \\
$W$ & Total available bandwidth at the BS \\
$\sigma_{i j}$ & Channel loss between BS $i$ and UE $j$ \\
\hline
\end{tabular}

Assuming $\lambda$ as the weighting factor between the 2 conflicting objectives, the optimization problem is formulated as follows:

$$
\begin{array}{ll}
\min _{\pi, x, \zeta} & \left(P_{c}+\lambda S\right) \\
\text { s.t. } \quad & \sum_{j=1}^{M} x_{i j} \leq N_{\mathrm{PRB}}, \quad \forall i \in \mathcal{B}, \\
& \sum_{i=1}^{N} \sum_{j=1}^{M} x_{i j}=M, \quad \forall j \in \mathcal{U}, \\
& \sum_{i=1}^{N} x_{i j}=1, \quad \forall j \in \mathcal{U}, \\
& c_{i j} \leq \frac{\pi_{i j} \cdot \sigma_{i j}}{P_{\mathrm{MIN} j}}, \quad \forall i \in \mathcal{B} \quad \forall j \in \mathcal{U}, \\
& c_{i j}-x_{i j} \geq 0, \quad \forall i \in \mathcal{B} \quad \forall j \in \mathcal{U}, \\
& \zeta_{i} \leq x_{i j}, \quad \forall j \in \mathcal{U} \quad \forall i \in \mathcal{B}, \\
& \sum_{j=1}^{M} \pi_{i j} \leq P_{\mathrm{MAX}}, \quad \forall i \in \mathcal{B} .
\end{array}
$$

Constraint $6 \mathrm{~b}$ is for the BS capacity limitation, since a BS cannot assign more than the available bandwidth elements $N_{\mathrm{PRB}}$. Constraints $6 \mathrm{c}$ and $6 \mathrm{~d}$ ensure that each UE must be covered by at least $1 \mathrm{BS}$ and can be connected to only $1 \mathrm{BS}$ at a time. Constraint $6 \mathrm{e}$ is the key for assuring the QoS: the binary variable $c_{i j}$ is equal to 0 if $\pi_{i j} \sigma_{i j} \leq P_{\mathrm{MIN} j}$; hence, for a given UE, constraint $6 \mathrm{e}$ will define the set of potential BSs that can provide the minimum received power, $P_{\mathrm{MIN} j}$. Then, introducing constraint $6 \mathrm{f}$, only one of the BSs in this set is selected. The activity status of a BS is linked to the user associations by constraint $6 \mathrm{~g}$. Finally, constraint $6 \mathrm{~h}$ sets the limit on the maximum BS transmission power.

The product of bounded variables in the objective function defines a general MIQP problem. It is well known that MIQP is NP-hard, trivially because it contains mixed-integer linear programming as a special case. In order to solve the optimization problem, the MIQP model has been built in an IBM ILOG CPLEX ${ }^{\circledR}$ solver, ${ }^{32}$ in which the optimal solution is obtained with an iterative method.

Since the optimization problem defines the linear combination of global power consumption and the number of BS switchings, the 2 entities have been normalized to be compared and weighted by $\lambda$.

A complete list of the considered parameters and their symbols is reported in Table 1. 

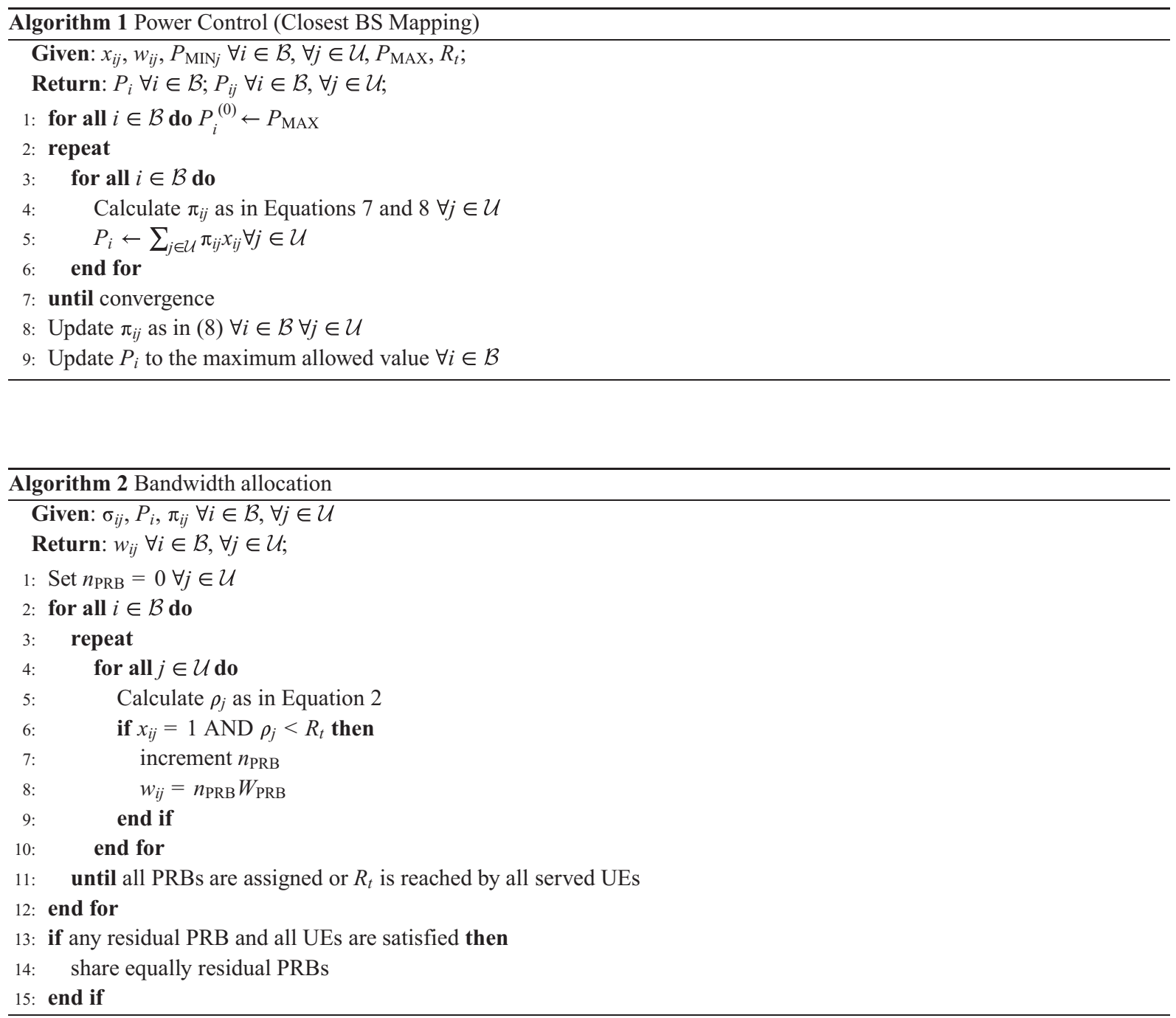

\section{4 | NETWORK OPTIMIZATION SOLUTIONS}

\section{1 | Power control and bandwidth adaptation}

Power control is a well-known solution to decrease the global energy consumption by acting on the reduction of intercell interference. ${ }^{15,21}$ In this work, a power control algorithm that is similar to the one presented in the works of Zander ${ }^{33}$ and Zander et $\mathrm{al}^{34}$ is considered, in order to extend that solution to a multichannel scenario with minimum and maximum power constraints. * As shown in Algorithm 1, this power control algorithm takes as input a UE-BS association and a bandwidth assignment for each UE and provides iteratively the optimum BS transmission power that can guarantee the target data rate for each UE. The proof of optimality can be found in the work of Zander. ${ }^{33}$ The core of the optimum power control algorithm is to calculate at each iteration $n$ the power transmitted by a BS to a certain UE as

$$
\pi_{i j}^{(n)}=\frac{w_{i j} 2^{\frac{R_{t}}{w_{i j}}}}{W \sigma_{i j}}\left(\sum_{k \in \mathcal{B}} P_{k}^{(n-1)}\left(1-x_{k j}\right) \sigma_{k j}+W N_{0}\right),
$$

where $R_{t}$ is the target data rate, ie, the QoS constraint for each UE. The initial condition is such that $\sum_{j} \pi_{i j}^{(0)}=P_{\mathrm{MAX}}$ for all $i \in \mathcal{B}$. Note that the power assigned to a BS $P_{i}$ cannot be greater than the maximum allowed power $P_{\mathrm{MAX}}$ : in that case, the power $P_{\mathrm{MAX}}$ is divided equally among each UE to indicate the UEs under outage. Moreover, the received power for each UE $j$

* For the proof of convergence in an iterative power control algorithm, please see the work of Zander et al ${ }^{34(\mathrm{pp} 163-171)}$. 
cannot be smaller than the sensitivity $P_{\mathrm{MINj}}$ : in that case, the power that is transmitted by a BS to a certain UE is adjusted by the following equation:

$$
\pi_{i j}=\max \left(\frac{P_{\mathrm{MIN} j}}{\sigma_{i j}} ; \pi_{i j}\right)
$$

Bandwidth assignment to each served UE is a task that can be solved in different ways by radio network operators depending on their policies. A radio network operator could give more priority to the UEs experiencing the best channel conditions to maximize the throughput. The assignment of bandwidth resources is crucial especially when the resources are limited. When an energy saving strategy is applied and the network capacity is reduced by putting a subset of BSs into sleep mode, a certain QoS level should be guaranteed to each served UE. Fairness between served UEs is also a key issue that can be introduced by giving more resources to the UEs experiencing the worst channel conditions. Such a solution enables the use of BEM to reduce the BS transmission power. Algorithm 2 presents a possible solution to assigning bandwidth in order to achieve fairness among UEs in terms of QoS, ie, user data rates.

\section{2 | Network configuration management optimization}

The fundamental approach of the optimization problem is to recognize the existence of multiple objectives, such as guaranteed rate for all the users, network power consumption, number of BS switchings, and number of simultaneously active BSs. A key assumption is that these objectives are not ordered, hence they are studied considering that in multicriteria optimization problems there are only subjectively optimal solutions. In order to minimize the power consumption in a cellular network, a first optimization strategy is proposed. The problem is iteratively solved for 3 variables: association between BS and UE, bandwidth assignment, and power allocation. In particular, a suitable BS-UE association allows saving power by decreasing the number of BSs that are not serving traffic and that can be deactivated or put in sleep mode. In order to limit the cost of switching operations, the number of power state transitions of BSs is also taken into account in the optimization process. By adopting the joint bandwidth and power allocation scheme, further gain is introduced, reducing the transmission power and decreasing the intercell interference. The deactivation and power reduction of the BSs are allowed only if no outage is introduced, ie, the target QoS is satisfied for each served UE. The optimization framework is composed of (1) the MIQP solver of the user association problem obtaining the optimized active subset of BSs, (2) the bandwidth allocation scheme, and (3) the power control algorithm, used to control the feasibility of the UE-to-BS mapping derived in (1) by identifying the eventual outages. The MIQP optimization refers to the problem formulated in Equations $6 \mathrm{a}$ to $6 \mathrm{~h}$. The output of this step is the mapping $x_{i j}$ between BSs and UEs, the power transmitted by each BS to each connected UE $\pi_{i j}$, and the set of active BSs $\zeta_{i}$. Then, in the second step, the bandwidth is allocated to each UE by the respective serving BSs. Bandwidth allocation is performed following Algorithm 2. The MIQP model is solved by an IBM ILOG CPLEX ${ }^{\circledR}$ solver. ${ }^{32}$ Since the model cannot manage directly the QoS for each UE because of its nonlinearity, 2 approaches are proposed to avoid any outage. Such approaches are (1) power consumption minimization assuming an interference-controlled scenario (MinPower) and (2) iterative power consumption and BS sleep-mode switching minimization to guarantee QoS (MinPower-QoS). The MinPower scenario assumes a good planning or a perfect intercell interference cancelation solution, but it could be also the reference condition for rural areas. If the rate of each user is only dependent on the signal-to-noise ratio, the only variable to be taken into account is the power received by the serving BS. On the other hand, if the interference cannot be neglected, the MinPower algorithm cannot guarantee the required QoS, as shown in Algorithm 3, and some outages could arise. Therefore, this algorithm represents an optimum lower bound for network optimization in terms of global power consumption. In order to avoid the data rate outages and reduce the impact of switching operations, MinPower-QoS is introduced in Algorithm 4. It combines the optimum power control and the MinPower approaches in an iterative framework. In particular, MinPower is executed to obtain the optimum set of active BSs, optimum mapping, and bandwidth assignment and to minimize power consumption, as well as the number of switch on/off operations, while the feasibility of this solution is controlled by the Power Control procedure, as shown in Algorithm 4. If some data rate outages occur, the power of the users that do not satisfy the target QoS is iteratively increased by a $\delta$ value to identify new active BS sets with better mapping. In order to design the network that aims at not only minimizing power consumption but also reducing the number of necessary BS switchings throughout a daily pattern of traffic demands, we characterize the attainable objective set of suboptimal solutions by computing a discrete set of $\lambda$ sample points. 

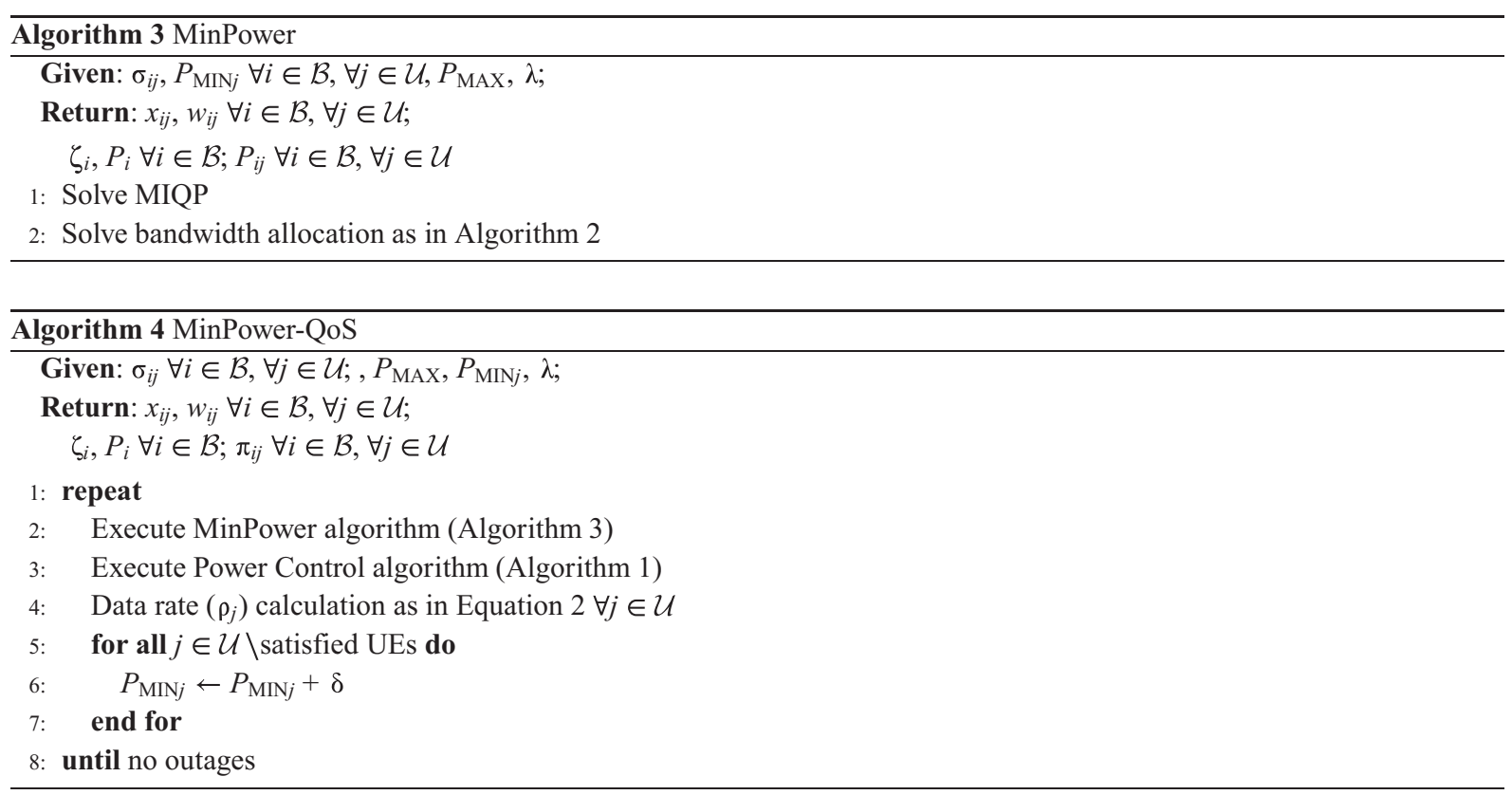

As $\lambda$ increases, the priority of reducing network operation and maintenance costs also increases.

\subsection{Algorithmic complexity}

In this section, the worst case performance of each algorithm is studied as a function of the number of users $M$ and the number of BSs $N$.

Power Control (Closest BS Mapping): The initialization step requires $N$ assignments. The algorithm cycles through the list of BSs, which requires $N$ iterations, with, at most, $M$ assignments and $M$ evaluations in each iteration. The final updates require $M N$ assignments. The algorithm is $\mathcal{O}\left(M N^{2}+M N\right)$.

Bandwidth allocation: The algorithm iterates $N$ times, and each stage requires $M$ evaluations and $M$ comparisons. The algorithm is $\mathcal{O}(M N)$.

MinPower: The MIQP problem is NP-hard. This means that, in the worst case, the solution time grows exponentially with the number of integer variables, but MIQP can easily be reformulated as a convex MIQP. This is true, for example, when all products are between a binary variable and a bounded variable. Unfortunately, the worst case complexity is still exponential, and the number of combinations necessary to enumerate and solve an optimization problem is problem dependent. CPLEX uses the branch-and-cut algorithm, based on branch and bound, to work in polynomial time when the number of constraints is small enough. ${ }^{32}$

MinPower-Qos: The dominant part of the algorithm is the execution of the MinPower algorithm; hence, the worst case complexity is still exponential. The second half of the algorithm, which involves Power Control and QoS target satisfaction, can be shown to be $\mathcal{O}\left(M N^{2}+M N\right)$. Once again, the use of preprocessing techniques in the CPLEX branch-and-cut algorithm can provide a solution to the optimization problem in polynomial time. ${ }^{32}$

\section{5 | SIMULATION RESULTS}

In order to evaluate the power consumption savings due to the BS sleep-mode mechanism and the transmission power adaptation, the proposed solution, namely, MinPower-QoS, is compared to upper bound and lower bound solutions. As an upper bound, the Closest BS Mapping is considered, adopting Algorithm 1, based on power control with closest BS mapping and equal bandwidth assignment to each UE. BSs that are not serving any UE are put on sleep mode. As a lower bound, the optimum energy saving solution MinPower is used. Moreover, to assess the trade-off between network energy consumption and the number of switching operations in MinPower-QoS, different $\lambda$ values have been considered. In Table 2, simulation parameters and the path loss model are reported. ${ }^{35}$ If a UE cannot achieve the data rate target, the minimum received power threshold, defined as $P_{\text {MIN } j}$ in constraint $6 \mathrm{e}$, is increased by $\delta=1 \mathrm{~dB}$ in each iteration of MinPower-QoS, solving the MIQP model with this new setting. Increasing 
TABLE 2 Simulation parameters ${ }^{35}$

\begin{tabular}{ll} 
Parameter & Value \\
\hline Deployment & $19 \mathrm{BS}$, hexagonal grid, wrap-around \\
\hline Intersite distance & $500 \mathrm{~m}$ \\
Path loss & $15.3+37.6 \log (d[\mathrm{~m}])$ (3GPP Typical Urban) \\
Shadow fading & std dev $8 \mathrm{~dB}$ \\
Indoor loss & $20 \mathrm{~dB}$ \\
\hline Bandwidth & $5 \mathrm{MHz}(25 \mathrm{PRBs})$ \\
Carrier frequency & $2 \mathrm{GHz}$ \\
Max BS $P_{T X}$ & $20 \mathrm{~W}$ \\
\hline UE sensitivity & $-90 \mathrm{dBm}$ \\
Noise PSD & $-174 \mathrm{dBm} / \mathrm{Hz}$ \\
Target user data rate & $512 \mathrm{Kbps}$ \\
Power consumption & $a=4.7 P_{0}=130 \mathrm{~W} P_{\text {sleep }}=13 \mathrm{~W}$ \\
\hline
\end{tabular}

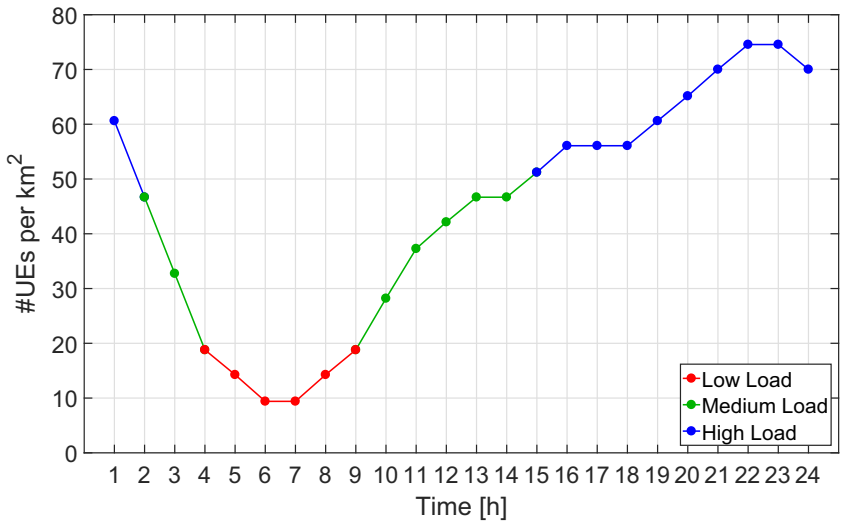

FIGURE 2 Daily traffic pattern

$P_{\mathrm{MINj}}$ by one unit at every step of the optimization process guarantees the fulfillment of QoS requirements for each network user. The number of active UEs that are randomly placed in the playground has been set considering the daily traffic profile in Figure 2. The trend of the UE requests shows a typical daily oscillation of the traffic that is consistent with the average profiles available in the literature. ${ }^{30}$ The maximum number of active UEs at busy hours in the considered area of $3 \mathrm{~km}^{2}$ is equal to 230, ie, $75 \mathrm{UEs} / \mathrm{km}^{2}$. It corresponds to the maximum number of UEs that can be managed by the Closest BS Mapping solution without any capacity outage, representing the maximum load of the cellular network. Three traffic load classes have been derived from the maximum traffic load, considering 20,50, and $75 \mathrm{UEs} / \mathrm{km}^{2}$ as peak values for low, medium, and high traffic loads, respectively. As shown by the research activities of the EARTH Project, ${ }^{30}$ the daily variation in the number of active users is analogous to the daily variation in traffic, and small-scale short-term evaluations fail to capture the EE of the entire network, since the load situation of a network varies radically over the course of a day and a week. Therefore, each single stage of the iterative process can represent a snapshot of the current network, and using average traffic statistics, the impact of user mobility can be neglected in the simulation scenario.

The results are obtained by statistical analysis of 50 simulation runs with a 95\% confidence interval. In Figure $3 \mathrm{~A}$, the UE satisfaction rate is depicted starting from the optimum MinPower solution as first iteration. From this figure, it is possible to note also the impact of the number of active UEs on the number of iterations before affording 0 outages. While MinPower experiences outages, MinPower-QoS converges to 0 outage performance after a number of iterations that depend on the number of UEs, ie, the network traffic load. Each iteration corresponds to a solution in the search space for the MIQP model, which stops when it reaches a minimum power solution with 0 outages. The cumulative transmission power versus the number of iterations is also depicted in Figure 3A. Active UEs are satisfied when mapping and received power allow to reach the target QoS. Among the feasible solutions, the one minimizing the global power consumption is chosen. Particularly, even if proper mapping is obtained by increasing the BS transmission power, this trend can be harmful for some UEs at the cell edge because of the interference level. Therefore, the cumulative transmission power of the active BSs is not only directly proportional to the 


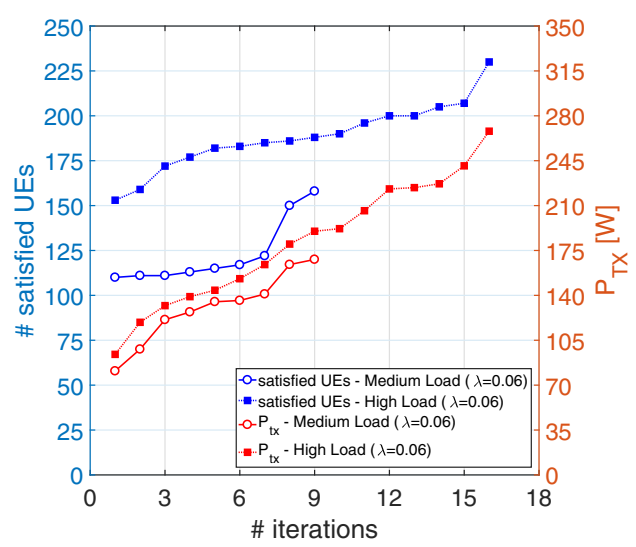

(A)

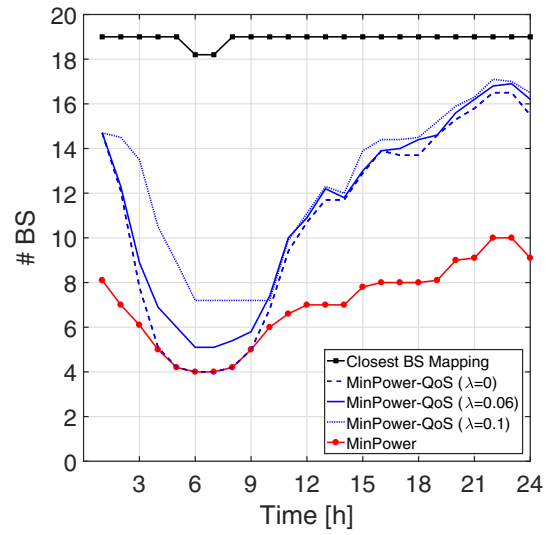

(B)

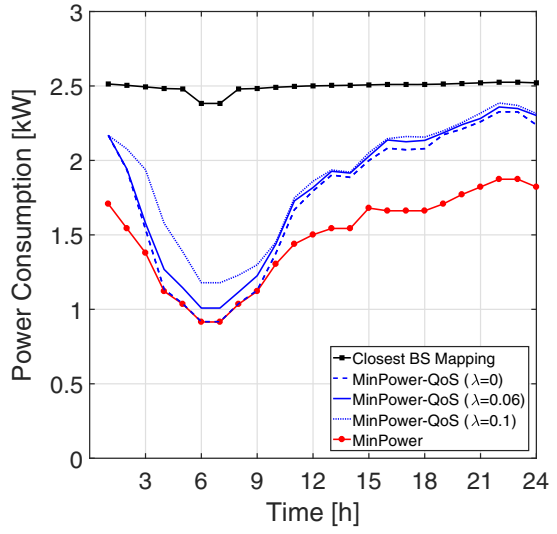

(C)

FIGURE 3 Simulation results. A, Satisfied UEs and cumulative transmission power of active BSs versus number of iterations in MinPower-QoS; $\mathrm{B}$, Active BSs versus time of day; $\mathrm{C}$, Global power consumption versus time of day

number of UEs, as shown in medium- and high-load cases, but also dependent on the current subset of active BSs at every step of the optimization process. On the other hand, a lower number of UEs require less iterations to reach the solution.

Figure 3B presents the comparison of the number of active BSs for MinPower-QoS, with different $\lambda$ values, with respect to the other solutions, ie, Closest BS Mapping and MinPower. From the figure, the behavior of the proposed solution is evident: when no optimization on the number of BS switchings is applied $(\lambda=0)$, the number of active BSs is the same as in the MinPower case in low-load hours. On the other hand, by increasing the weight of BS switchings in the optimization process, the number of active BSs ends up being more stable. Because of the QoS requirements, the slope of MinPower-QoS is higher than MinPower: as expected, MinPower-QoS converges to Closest BS Mapping when the number of active UEs increases at busy hours. This result is enforced by Figure $3 \mathrm{C}$ where total power consumption versus time is depicted. It is interesting to see that in the interference-limited scenario under high load, MinPower-QoS can still have power savings over the Closest BS Mapping algorithm due to its flexibility in user association and bandwidth allocation. On the other hand, for the minimum load demand, the performance of MinPower-QoS is close to the energy consumption optimum lower bound represented by MinPower. More in detail, the impact of the weighting factor $\lambda$ in the MinPower-QoS optimization process can be highlighted. The solution with an intermediate value of $\lambda=0.06$ has a remarkable performance from the power consumption perspective, by maintaining a good compromise in terms of power saving during peak and off-peak hours. Such behavior can be explained by a more flexible management of the radio resources, which allows a lower number of active BSs and lower transmission power. On the other hand, when the weight of $\lambda$ increases, the adaptability of the network configuration is reduced in terms of switching operations and the total power consumption inevitably increases, especially in low-load hours. More details about this trend are provided in Figure 4. The results achieved by MinPower-QoS, in terms of daily energy consumption and daily number of BS sleep-mode switchings, are shown for the set of discrete values $\lambda \in\{0,0.01,0.02,0.04,0.06,0.08,0.1\}$. When $\lambda=0$, the optimization framework obtains the best energy saving solution at the expense of a greater number of BS sleep-mode switchings. The introduction of the multiobjective optimization approach with weighting factor $\lambda$ on the other side drastically reduces the number of sleep-mode transitions, consuming more energy with higher values of $\lambda$. In particular, the optimal trade-off is guaranteed by the $\lambda=0.06$ case, which consumes $3 \%$ more energy to the benefit of $70 \%$ less daily number of BS switchings with respect to the $\lambda=0$ case. Since the optimization model is based on reaching the best solution with 0 outages, it is important to remind that the introduction of $\lambda$ does not alter the satisfied UE target, while searching for the best attainable BS subset, as shown in Figure 3A. Moreover, in Figure 5, the running time for the MinPower-QoS algorithm is plotted as a function of the number of users with 3 different values of $\lambda$. Measurements were performed on a system featuring an Intel Centrino Core 2 Duo E6600 CPU running at $2.20 \mathrm{GHz}$ and 4-GB RAM. The proposed method requires tens of seconds in medium-to-high traffic load cases. Although the running time increases with the growth in user population, the impact of the optimization process in terms of reduced number of BS switchings moderately affects the performances with higher values of $\lambda$.

In order to evaluate the overall throughput performance of the proposed solution, Figure 6 shows the results obtained in terms of daily power consumption and aggregate daily throughput. While MinPower is able to heavily reduce the amount of consumed power, the lack of QoS requirements causes a huge loss in terms of the overall throughput achieved by users. On the other hand, MinPower-QoS can obtain an acceptable throughput performance, holding up to the Closest BS Mapping reference case and delivering major energy savings at the same time. 


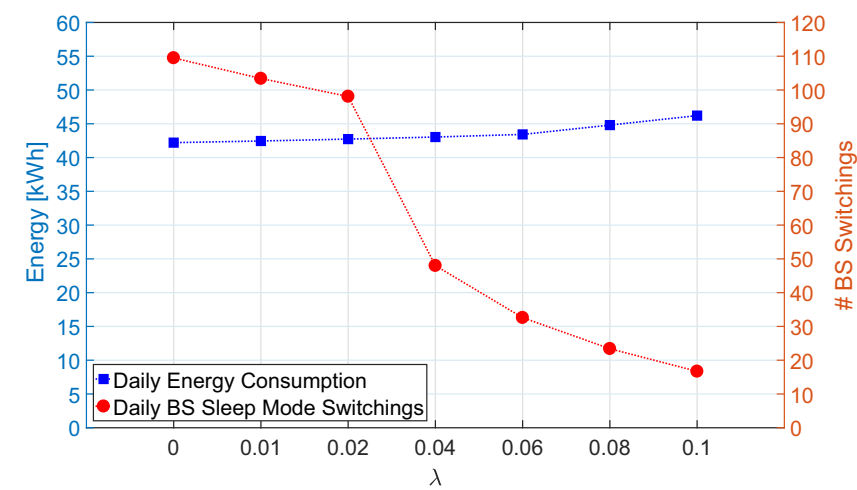

FIGURE 4 Daily energy consumption versus number of BS switchings in MinPower-QoS

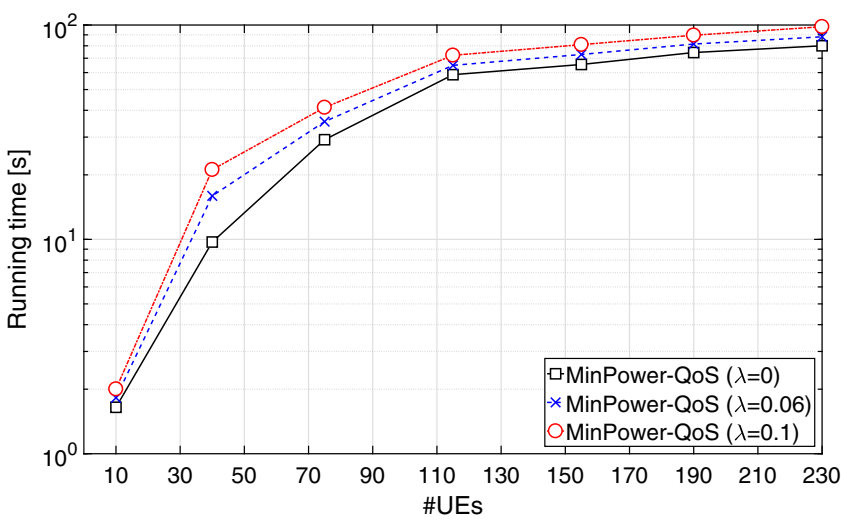

FIGURE 5 Running time as a function of the number of users in MinPower-QoS

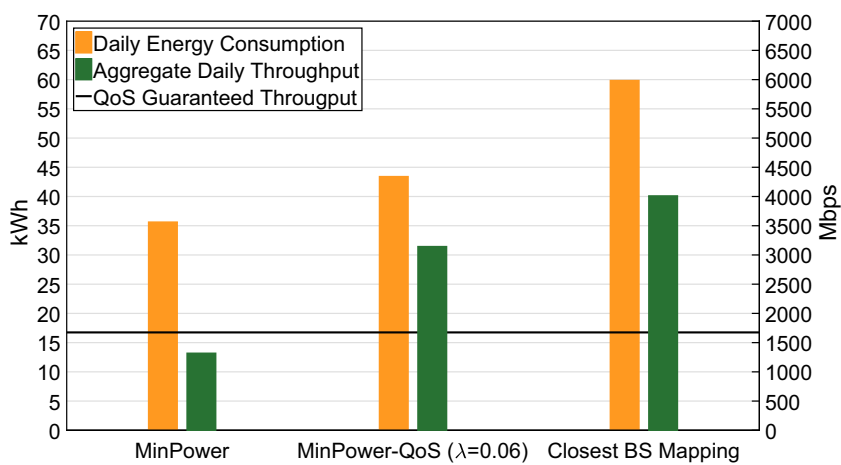

FIGURE 6 Daily energy consumption and aggregate throughput

In Figure 7, the optimization solutions obtained by MinPower-QoS are divided into 3 subsets, corresponding to low, medium, and high traffic load periods, as highlighted in Figure 2. The introduction of the weighting factor $\lambda$ in the optimization process allows to heavily reduce the number of switching operations during the day, particularly at off-peak hours, when the network degree of adaptability is higher. Note that with lower values of $\lambda$, the power consumption sligthly changes until no optimization is applied in terms of switching operations $(\lambda=0)$; on the other hand, employing the highlighted optimal $\lambda$ values, the hourly number of BS switchings drops below one-half of the peak value in the low-load case and below one-third of the peak value in the medium- and high-load cases. Consequently, the optimal network configuration can be obtained, affording the coverage and QoS requirements with a minimum number of BS switchings. 


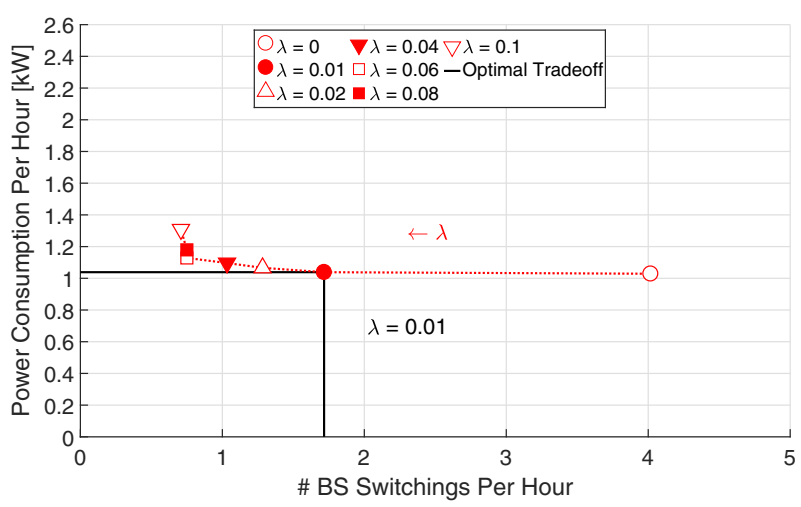

(A)

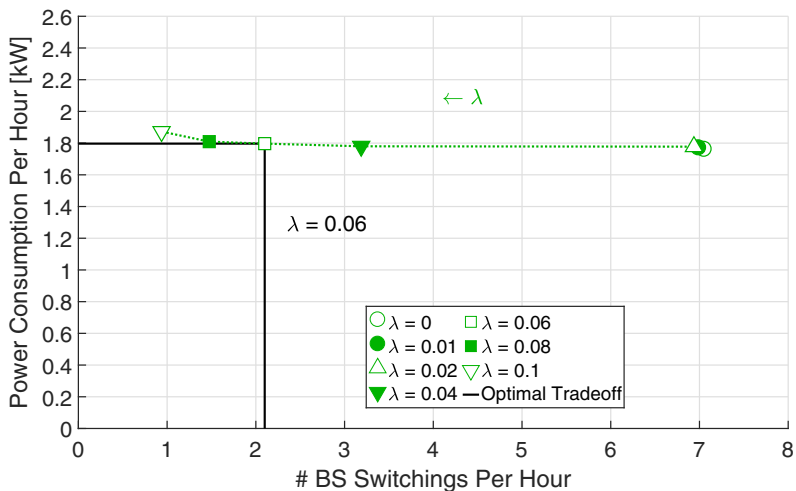

(B)

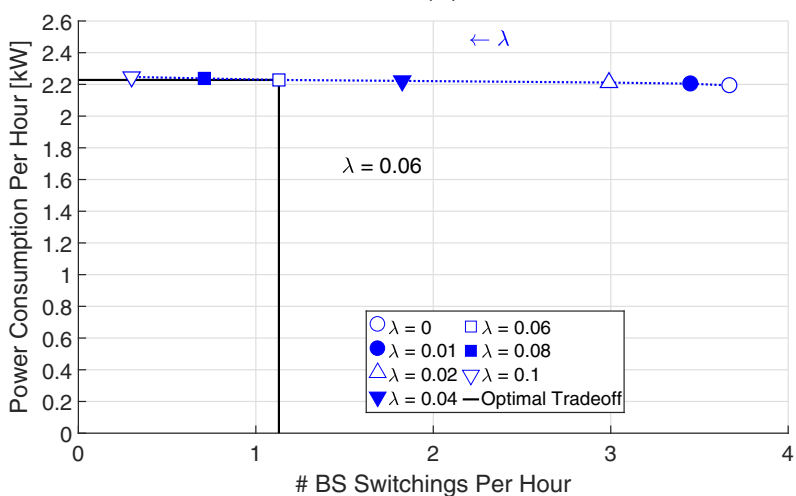

(C)

FIGURE 7 Optimal trade-off between power consumption and number of BS switchings. A, Low-load hours; B, Medium-load hours;

C, High-load hours

Such a solution is able to reduce the signaling and handover traffic overhead related to the joint BS on/off switching and user association strategy, as well as the impact on operations and maintenance activities, which involve operational performance, monitoring, and control of site operations. Moreover, it can be seen that the impact of the $\lambda$ factor is more evident when the network is in low-load conditions: by adopting the minimum weight value $\lambda=0.01$ in the optimization process, the power savings obtained by MinPower-QoS can be preserved, without increasing the number of active BSs. Conversely, to obtain an optimal performance for all the network configuration requirements, in medium- and high-load cases, a higher value of $\lambda$ is necessary: for $\lambda=0.06$, not only is the number of BS switchings highly reduced but also are the number of active BSs and the network power consumption optimized at busy hours with respect to the $\lambda=0$ reference case.

Considering the trade-off between energy consumption and network performance, the optimization process of MinPower-QoS is shown to be particularly effective when the network is not heavily loaded, as depicted in Figure 8A: the proposed solution, in fact, guarantees comparable energy use as in the MinPower strategy, without compromising the network throughput performance. Moreover, the flexible management of user association and bandwidth distribution of MinPower-QoS allows to greatly exceed the QoS target throughput, even in heavily loaded network scenarios, as shown in Figure 8B,C. 


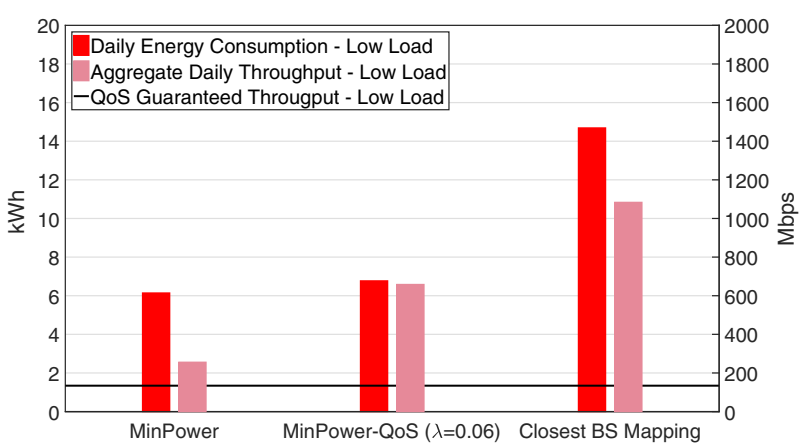

(A)

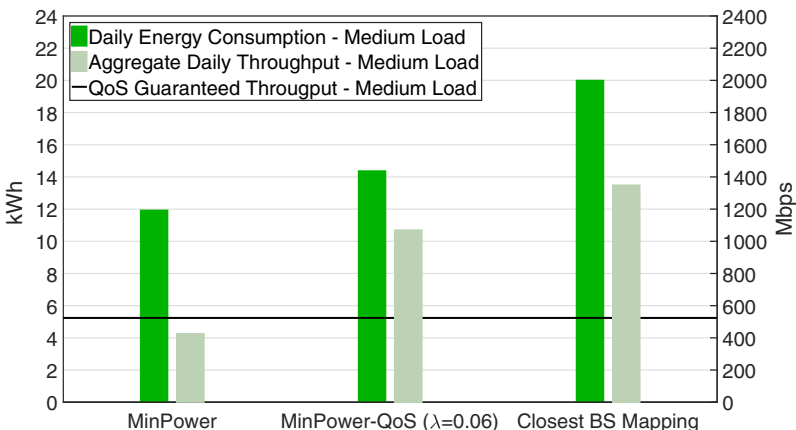

(B)

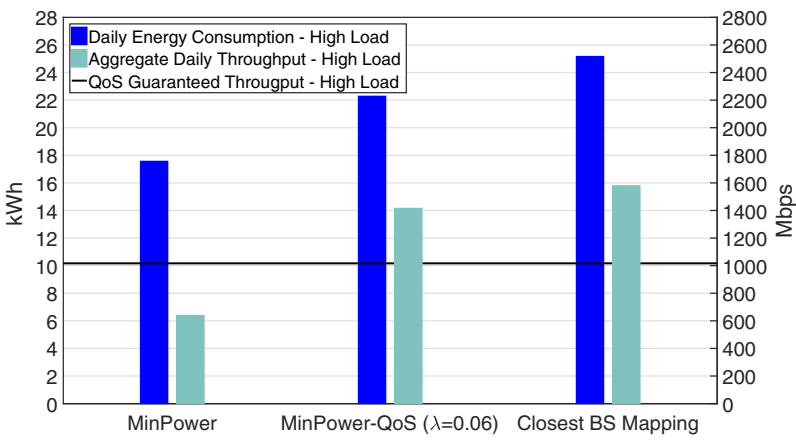

(C)

FIGURE 8 Throughput and energy consumption analysis. A, Low-load hours; B, Medium-load hours; C, High-load hours

\section{6 | CONCLUSION}

In this paper, a novel multiobjective optimization framework is developed, which allows to study and solve the network power consumption minimization problem, guaranteeing QoS target requirements and minimum number of BS switchings. The results for interference-limited scenarios and average daily traffic loads are shown. By putting the cells into sleep mode, up to 60\% power savings can be achieved at off-peak hours with respect to the basic scheme. Furthermore, the proposed MinPower-QoS methodology affords performance very close to the optimum solution, particularly for low-traffic-load scenarios. By optimizing the network configuration, a 70\% reduction in BS switch on/off operations can be reached in a day with 3\% more energy expense. More in detail considering daily traffic variations, significant savings can be obtained when the traffic is below $35 \%$ of the maximum load. Above that level, in medium-to-high traffic load, $25 \%$ and $10 \%$ power savings are still obtainable, but other EE features can be preferred instead of deep sleep. The combination of different energy saving features and additional optimization objectives is considered as a future work.

\section{REFERENCES}

1. Prakash S, Baron Y, Liua R, Proske M, Schlösser A. Study on the practical application of the new framework methodology for measuring the environmental impact of ICT_Cost/benefit analysis. European Commission; 2014.

2. Saker L, Elayoubi S. Sleep mode implementation issues in green base stations. Paper presented at: IEEE 21st International Symposium on Personal, Indoor and Mobile Radio Communications (PIMRC); 2010; Istanbul, Turkey. https://doi.org/10.1109/PIMRC.2010.5671926 
3. Saker L, Elayoubi SE, Chahed T. Minimizing energy consumption via sleep mode in green base station. Paper presented at: IEEE Wireless Communications and Networking Conference (WCNC); 2010; Sydney, Australia. https://doi.org/10.1109/WCNC.2010.5506413

4. Morosi S, Piunti P, Del Re E. Improving cellular network energy efficiency by joint management of sleep mode and transmission power. Paper presented at: 24th Tyrrhenian International Workshop on Digital Communications—Green ICT (TIWDC); 2013; Genoa, Italy. https://doi.org/ 10.1109/TIWDC.2013.6664207

5. Marsan M, Chiaraviglio L, Ciullo D, Meo M. Switch-off transients in cellular access networks with sleep modes. Paper presented at: IEEE International Conference on Communications (ICC); 2011; Kyoto, Japan. https://doi.org/10.1109/iccw.2011.5963559

6. Frenger P, Moberg P, Malmodin J, Jading Y, Godor I. Reducing energy consumption in LTE with cell DTX. Paper presented at: IEEE 73rd Vehicular Technology Conference (VTC Spring); 2011; Budapest, Hungary. https://doi.org/10.1109/VETECS.2011.5956235

7. Hedayati M, Amirijoo M, Frenger P, Moe J. Reducing energy consumption through adaptation of number of active radio units. Paper presented at: IEEE 73rd Vehicular Technology Conference (VTC Spring); 2011; Budapest, Hungary. https://doi.org/10.1109/VETECS.2011.5956486

8. Wang R, Thompson J, Haas H, Grant P. Sleep mode design for green base stations. IET Commun. 2011;5(18):2606-2616. https://doi.org/10. 1049/ietcom.2011.0104

9. Chen T, Yang Y, Zhang H, Kim H, Horneman K. Network energy saving technologies for green wireless access networks. IEEE Wirel Commun. 2011;18(5):30-38. https://doi.org/10.1109/MWC.2011.6056690

10. Videv S, Haas H. Energy-efficient scheduling and bandwidth-energy efficiency trade-off with low load. Paper presented at: IEEE International Conference on Communications (ICC); 2011; Kyoto, Japan. https://doi.org/10.1109/icc.2011.5962571

11. Marsan MA, Chiaraviglio L, Ciullo D, Meo M. Multiple daily base station switch-offs in cellular networks. Paper presented at: Fourth International Conference on Communications and Electronics (ICCE); 2012; Hue, Vietnam. https://doi.org/10.1109/CCE.2012.6315906

12. Marsan MA, Chiaraviglio L, Ciullo D, Meo M. On the effectiveness of single and multiple base station sleep modes in cellular networks. Comput Netw. 2013;57(17):3276-3290. https://doi.org/10.1016/j.comnet.2013.07.016

13. Tabassum H, Siddique U, Hossain E, Hossain MJ. Downlink performance of cellular systems with base station sleeping, user association, and scheduling. IEEE Trans Wirel Commun. 2014;13(10):5752-5767. https://doi.org/10.1109/TWC.2014.2336249

14. Wu J, Zhang Y, Zukerman M, Yung EKN. Energy-efficient base-stations sleep-mode techniques in green cellular networks: a survey. IEEE Commun Surveys Tuts Second Quarter. 2015;17(2):803-826. https://doi.org/10.1109/COMST.2015.2403395

15. Venturino L, Risi C, Zappone A, Buzzi S. Green joint user scheduling and power control in downlink multi-cell OFDMA networks. Paper presented at: Future Network and Mobile Summit (FutureNetworkSummit); 2013; Lisboa, Portugal.

16. Wong CY, Cheng R, Lataief K, Murch R. Multiuser OFDM with adaptive subcarrier, bit, and power allocation. IEEE J Sel Areas Commun. 1999;17(10):1747-1758. https://doi.org/10.1109/49.793310

17. Yildiz A, Girici T, Yanikomeroglu H. A pricing based algorithm for cell switching off in green cellular networks. Paper presented at: IEEE 77th Vehicular Technology Conference (VTC Spring); 2013; Dresden, Germany. https://doi.org/10.1109/VTCSpring.2013.6691841

18. Son K, Kim H, Yi Y, Krishnamachari B. Base station operation and user association mechanisms for energy-delay tradeoffs in green cellular networks. IEEE J Sel Areas Commun. 2011;29(8):1525-1536. https://doi.org/10.1109/JSAC.2011.110903

19. Piunti P, Cavdar C, Morosi S, Teka K, Del Re E, Zander J. Energy efficient adaptive cellular network configuration with QOS guarantee. Paper presented at: IEEE International Conference on Communications (ICC); 2015; London, UK. https://doi.org/10.1109/ICC.2015.7248300

20. Holtkamp H, Auer G, Bazzi S, Haas H. Minimizing base station power consumption. IEEE J Sel Areas Commun. 2014;32(2):297-306. https:// doi.org/10.1109/JSAC.2014.141210

21. Huang X, Ge X, Wang Y, Li F, Zhang J. Energy-efficient binary power control with bit error rate constraint in MIMO-OFDM wireless communication systems. Paper presented at: IEEE Vehicular Technology Conference (VTC Fall); 2012; Quebec City, Canada. https://doi.org/10.1109/ VTCFall.2012.6398994

22. Xiao X, Tao X, Lu J. QoS-guaranteed energy-efficient power allocation in downlink multi-user MIMO-OFDM systems. Paper presented at: IEEE International Conference on Communications (ICC); 2014; Sydney, Australia. https://doi.org/10.1109/ICC.2014.6883937

23. Ren Z, Chen S, Hu B, Ma W. Energy-efficient resource allocation in downlink OFDM wireless systems with proportional rate constraints. IEEE Trans Veh Technol. 2014;63(5):2139-2150. https://doi.org/10.1109/TVT.2014.2311235

24. Bjornson E, Jorswieck E, Debbah M, Ottersten B. Multiobjective signal processing optimization: the way to balance conflicting metrics in $5 \mathrm{G}$ systems. IEEE Signal Process Mag. 2014;31(6):14-23. https://doi.org/10.1109/MSP.2014.2330661

25. Chen Y, Zhang S, Xu S, Li G. Fundamental trade-offs on green wireless networks. IEEE Commun Mag. 2011;49(6):30-37. https://doi.org/10. 1109/MCOM.2011.5783982

26. Oh E, Krishnamachari B. Energy savings through dynamic base station switching in cellular wireless access networks. Paper presented at: IEEE Global Telecommunications Conference (GLOBECOM); 2010; Miami, Florida, USA. https://doi.org/10.1109/GLOCOM.2010.5683654

27. Gonzalez DG, Yanikomeroglu H, Garcia-Lozano M, Boque SR. A novel multiobjective framework for cell switch-off in dense cellular networks. Paper presented at: IEEE International Conference on Communications (ICC); 2014; Sydney, Australia. https://doi.org/10.1109/ICC. 2014.6883722

28. Piunti P, Dolfi M, Morosi S, Jayousi S, Del Re E. Performance evaluation of an energy efficient RRM strategy in heterogeneous cellular networks. Paper presented at: IEEE 25th International Symposium on Personal, Indoor and Mobile Radio Communications (PIMRC); 2014; Washington DC, USA.

29. Alam AS, Dooley LS. A scalable multimode base station switching model for green cellular networks. Paper presented at: IEEE Wireless Communications and Networking Conference (WCNC); 2015; New Orleans, LA, USA. https://doi.org/10.1109/WCNC.2015.7127585

30. Auer G, Blume O, Giannini V, et al. EARTH project deliverable D2.3: energy efficiency analysis of the reference systems, areas of improvements and target breakdown, INFSO-ICT-247733 EARTH; 2012. 
31. Auer G, Giannini V, Desset C, et al. How much energy is needed to run a wireless network? IEEE Wirel Commun. 2011;18(5):40-49. https://doi. org/10.1109/MWC.2011.6056691

32. Bliek1ú C, Bonami P, Lodi A. Solving mixed-integer quadratic programming problems with IBM-CPLEX: a progress report; 2014.

33. Zander J. Performance of optimum transmitter power control in cellular radio systems. IEEE Trans Veh Technol. 1992;41(1):57-62. https://doi. org/10.1109/25.120145

34. Zander J, Kim SL, Almgren M, Queseth O. Radio Resource Management for Wireless Networks. Norwood, MA: Artech House, Inc; 2001.

35. Further advancements for E-UTRA physical layer aspects v.9.0.0. 3GPP TR 36.814; 2010.

How to cite this article: Dolfi M, Cavdar C, Morosi S, Piunti P, Zander J, Del Re E. On the trade-off between energy saving and number of switchings in green cellular networks. Trans Emerging Tel Tech. $2017 ; \mathrm{e} 3193$. https://doi.org/10.1002/ett.3193 